\title{
About One-Dimensional Conservative Systems with Position Depending Mass
}

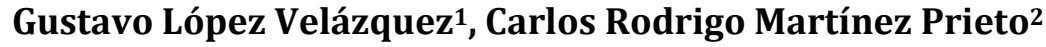 \\ ${ }^{1}$ Departamento de Fsica, Universidad de Guadalajara, Guadalajara, México \\ ${ }^{2}$ Instituto Tecnológico y Estudios Superiores de Occidente, Tlaquepaque, México \\ Email: gulopez@udgserv.cencar.udg.mx, CARLOSR@iteso.mx
}

Received 27 April 2014; revised 24 May 2014; accepted 18 June 2014

Copyright (C) 2014 by authors and Scientific Research Publishing Inc.

This work is licensed under the Creative Commons Attribution International License (CC BY).

http://creativecommons.org/licenses/by/4.0/

(c) (i) Open Access

\section{Abstract}

For a one-dimensional conservative system with position depending mass, one deduces consistently a constant of motion, a Lagrangian, and a Hamiltonian for the nonrelativistic case. With these functions, one shows the trajectories on the spaces $(x, v)$ and $(x, p)$ for a linear position depending mass. For the relativistic case, the Lagrangian and Hamiltonian cannot be given explicitly in general. However, we study the particular system with constant force and mass linear dependence on the position where the Lagrangian can be found explicitly, but the Hamiltonian remains implicit in the constant of motion.

\section{Keywords}

\section{Mass Variable Systems, Conservative System, Position Depending Mass}

\section{Introduction}

Position depending mass systems have been relevant since the foundation of the classical mechanics and modern physics [1]-[5] (see reference there in). Actually, the interest for these type of problems has grown in modern physics due to fabrication of ultra thin semiconductors [6] [7], inhomogeneous crystals [8], quantum dots [9], quantum liquids [10], and neutrino mass oscillations [11] [12]. We also need to mention that this topic is important due to its relation with the foundation of the classical mechanics [13], and its not invariance under Galileo or Poincar-Lorentz transformations [13] [14]. Most of the approaches dealing with position depending mass problems use an intuitive way to write down a Lagrangian or Hamiltonian for the system, and then solve the corresponding equations [15] [16]. In this paper, one obtains a constant of motion, a Lagrangian, and a Hamiltonian in a consistent way for conservative nonrelativistic systems and study the harmonic oscillator with position depending mass as an example. For relativistic systems we point out the difficulty to get the same func- 
tions.

\section{Dynamical Functions}

A non relativistic conservative system with position depending mass is described by Newton’s equation

$$
\frac{\mathrm{d}}{\mathrm{d} t}(m(x) \dot{x})=F(x)
$$

where $\dot{x}$ denotes the velocity of the body with position depending mass $m(x)$, and with a force $F(x)$ acting on it. This problem can be written as the dynamical system

$$
\dot{x}=v, \quad \dot{v}=\frac{F(x)}{m(x)}-\frac{m_{x}}{m} v^{2},
$$

where $m_{x}$ is the differentiation of the mass with respect the position. A constant of motion of this system is a function $K=K(x, v)$ [17] [18] which satisfies the following first order partial differential equation

$$
v \frac{\partial K}{\partial x}+\frac{1}{m(x)}\left[F(x)-m_{x} v^{2}\right] \frac{\partial K}{\partial v}=0 .
$$

This equation can be solved by the characteristics method. The equations for its characteristics curves are

$$
\frac{\mathrm{d} x}{v}=\frac{m(x) \mathrm{d} v}{F(x)-m_{x} v^{2}}=\frac{\mathrm{d} K}{0} .
$$

From the last term, one knows that the solution of (3) must be of the form

$$
K(x, v)=G(C(x, v)),
$$

where $C(x, v)$ is the characteristic curve obtained from the first two terms of (4), and being $G$ and arbitrary function. This characteristic curve can be found arranging these two terms of the form

$$
m(x) v \frac{\mathrm{d} v}{\mathrm{~d} x}=F(x)-m_{x} v^{2},
$$

and defining a new variable, $\xi=v^{2}$, to get the equation

$$
m(x) \frac{\mathrm{d} \xi}{\mathrm{d} x}+2 m_{x} \xi=2 F(x)
$$

which can readily be integrated to obtain the characteristic curve

$$
C(x, v)=\frac{m^{2}(x)}{2} v^{2}-\int m(x) F(x) \mathrm{d} x .
$$

Choosing the initial conditions $x(0)=0, v(0) \neq 0$, and $m(0)=m_{0}$, and selecting the functionality $G(C(x, v))=C(x, v) / m_{0}$, the constant of motion is

$$
K(x, v)=\frac{m^{2}(x)}{2 m_{0}} v^{2}+V_{e f f}(x),
$$

where $V_{\text {eff }}$ is the effective potential due to the position depending mass,

$$
V_{\text {eff }}(x)=-\frac{1}{m_{0}} \int m(x) F(x) \mathrm{d} x,
$$

and this constant of motion has the right expression when constant mass is considered. Using now the known expression [19]-[21] to get the Lagrangian from a constant of motion, 


$$
L(x, v)=v \int \frac{K(x, \xi)}{\xi^{2}} \mathrm{~d} \xi
$$

The Lagrangian, generalized linear momentum and Hamiltonian are given by

$$
\begin{gathered}
L(x, v)=\frac{m^{2}(x)}{2 m_{0}} v^{2}-V_{\text {eff }}(x), \\
p(x, v)=\frac{m^{2}(x)}{m_{0}} v,
\end{gathered}
$$

and

$$
H(x, p)=\frac{m_{0}}{2 m^{2}(x)} p^{2}+V_{e f f}(x) .
$$

The above expression for the dynamical functions show that there are two main modifications from the usual expression when position depending mass is considered. Firstly, an effective potential is created which depends on $m(x)$ (10). Secondly, the kinetic energy like term is not of the form $p^{2} / 2 m(x)$ but it is of the form $m_{0} p^{2} / 2 m^{2}(x)$. These two modification are really important to deal correctly with an specific mass position depending conservative problem.

\section{Harmonic Oscillator $m(x)$}

Consider the harmonic oscillator, $F(x)=-k x$, with a linear position depending mass, $m(x)=m_{0}+m_{1} x$. Thus, the effective potential, constant of motion, and Hamiltonian are given by

$$
\begin{gathered}
V_{\text {eff }}(x)=\frac{k}{2} x^{2}+\frac{m_{1} k}{3 m_{0}} x^{3}, \\
K(x, v)=\frac{\left(m_{0}+m_{1} x\right)^{2}}{2 m_{0}} v^{2}+\frac{k}{2} x^{2}+\frac{m_{1} k}{3 m_{0}} x^{3},
\end{gathered}
$$

and

$$
H(x, p)=\frac{m_{0}}{2\left(m_{0}+m_{1} x\right)^{2}} p^{2}+\frac{k}{2} x^{2}+\frac{m_{1} k}{3 m_{0}} x^{3} .
$$

Figure 1(a) and Figure 1(b) show the trajectories on the spaces $(x, v)$ and $(x, p)$ generated by the constant of motion and the Hamiltonian above with $m_{0}=200$ and for $m_{1}>0 \quad\left(m_{0}=200 ; m_{1}=0\right.$ (1), $m_{1}=5$ (2), $m_{1}=10$ (3), $m_{1}=20$ (4)). Figure 2(a) and Figure 2(b) show also the trajectories on those spaces with $m_{0}=1000$ and $m_{1}<0\left(m_{1}=0\right.$ (1), $m_{1}=-1$ (2), $m_{1}=-1.5$ (3), $m_{1}=-2$ (4)). From Figure 2(a) one notes a singular behavior in the velocity which comes from (16) and does not appears in the Hamiltonian formulation (17).

Note that due to relation (10), it is not possible to know the potential (effective) without the acknowledge of the position depending mass previously.

\section{Relativistic Case}

The relativistic motion [22] of a body with position depending mass is not invariant under Poincaré-Lorentz transformation [13], but it still can be described by the equation

$$
\frac{\mathrm{d}}{\mathrm{d} t}(\gamma m(x) \dot{x})=F(x), \quad \gamma=\left(1-\frac{\dot{x}^{2}}{c^{2}}\right)^{-1 / 2},
$$

where $c$ is the speed of light, and it can be written as a Newton's equation with a velocity depending force of 

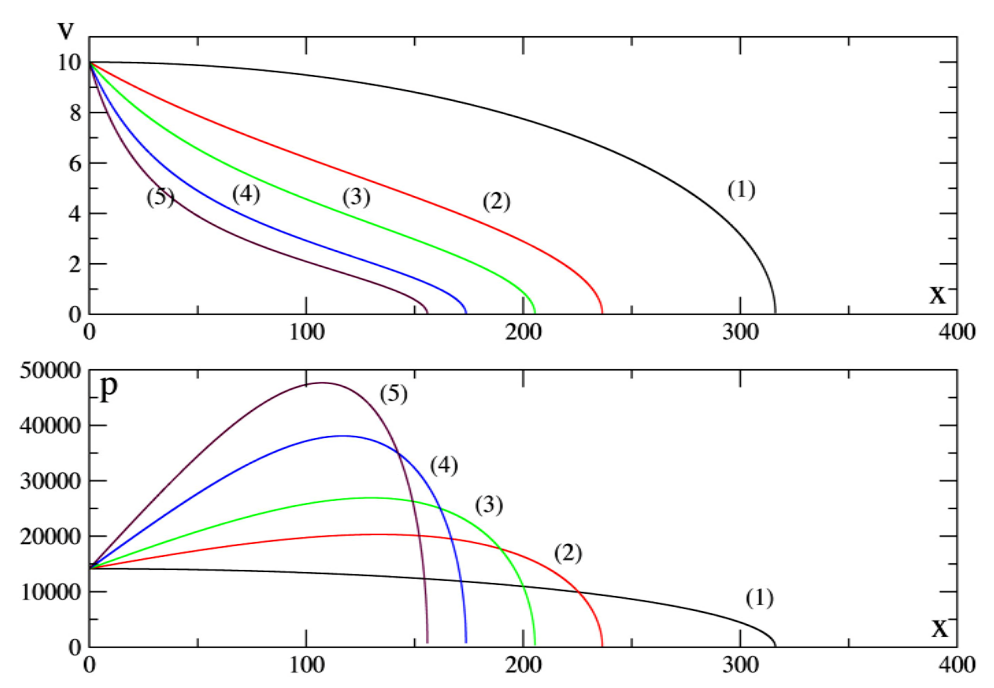

Figure 1. Trajectories for $m_{1}>0$.
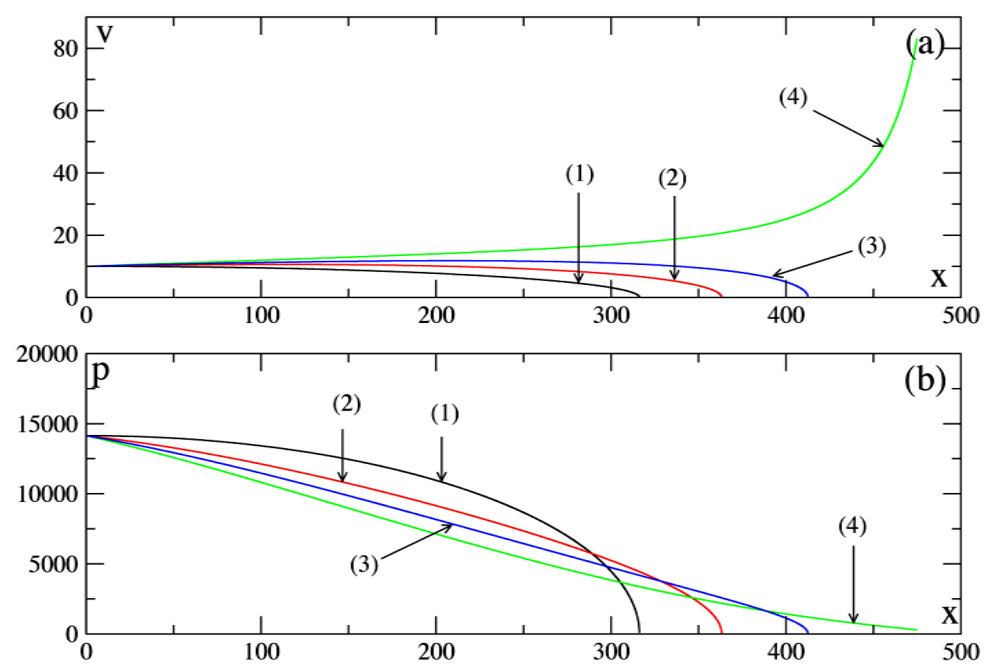

Figure 2. Trajectories for $m_{1}<0$.

the form

$$
m(x) \frac{\mathrm{d}^{2} x}{\mathrm{~d} t^{2}}=\left[F(x)-\frac{\dot{x}^{2} m_{x}}{\sqrt{1-(\dot{x} / c)^{2}}}\right]\left(1-\frac{\dot{x}^{2}}{c^{2}}\right)^{3 / 2} .
$$

In turns, this equation defines the dynamical system

$$
\dot{x}=v,
$$

$$
\dot{v}=\frac{1}{m(x)}\left[F(x)-\gamma v^{2} m_{x}\right] \gamma^{-3} .
$$

As before, a constant of motion of this autonomous system is a function $K=K(x, v)$ satisfying the equation

$$
v \frac{\partial K}{\partial x}+\frac{1}{m(x)}\left[F(x)-\gamma v^{2} m_{x}\right] \gamma^{-3} \frac{\partial K}{\partial v}=0 .
$$


The equations for the characteristics are

$$
\frac{\mathrm{d} x}{v}=\frac{m(x) \mathrm{d} v}{\left[F(x)-\gamma v^{2} m_{x}\right]\left(1-\frac{v^{2}}{c^{2}}\right)^{3 / 2}}=\frac{\mathrm{d} K}{0} .
$$

Thus, this constant of motion is an arbitrary function of the characteristic obtained from the first two term of this expression, $K=G(C(x, v))$, and from the first two terms one gets the following equation in terms of the variable $\eta=v^{2} / c^{2}$,

$$
\frac{m(x) c^{2}}{2} \frac{\mathrm{d} \eta}{\mathrm{d} x}=\left[F(x)-\frac{m_{x} c^{2} \eta}{\sqrt{1-\eta}}\right](1-\eta)^{3 / 2} .
$$

The constant of integration of this equation will represent the characteristic curve $C(x, \eta)$. Of course, in general the solution of this equation is not expressed in close form. This means that the Lagrangian and the Hamiltonian of the system can not be found in general. However, there is a particular case where one can do something analytically, and this case consists of having a constant force with mass linearly dependence on the position.

\subsection{Constant Force with $m(x)=m_{0}+m_{1} x$}

In this particular case, one has that $m_{x}=m_{1}$ and $F(x)=F=$ constant, and the variables can be separated for the integration in (24), bringing about the characteristic curve

$$
C(x, \eta)=\int \frac{\mathrm{d} \eta}{\left(F-\frac{m_{1} \eta}{\sqrt{1-\eta}}\right)(1-\eta)^{3 / 2}}-\frac{2}{c^{2} m_{1}} \ln \left(m_{0}+m_{1} x\right) .
$$

By selecting the constant of motion as

$$
K(x, \eta)=\frac{F m_{0} c^{2}}{2} C(x, \eta)+\frac{F a}{m_{1}} \ln m_{0}-m_{0} c^{2},
$$

that is,

$$
K(x, \eta)=\frac{m_{0} c^{2}}{2} \int \frac{\mathrm{d} \eta}{\left(1-\frac{m_{1} \eta}{F \sqrt{1-\eta}}\right)(1-\eta)^{3 / 2}}-\frac{m_{0} F}{m_{1}} \ln \left(m_{0}+m_{1} x\right)+\frac{F m_{0}}{m_{1}} \ln m_{0}-m_{0} c^{2},
$$

one has the following limit

$$
\lim _{m_{1} \rightarrow 0} K(x, \eta)=\gamma m_{0} c^{2}-F x-m_{0} c^{2},
$$

which corresponds to the relativistic case of constant mass. Now, considering the condition

$$
\left|\frac{m_{1} \eta}{F \sqrt{1-\eta}}\right| \leq 1
$$

one can write the constant of motion as [23]

$$
\begin{aligned}
K(x, \eta)= & \gamma m_{0} c^{2}-F x-m_{0} c^{2}+m_{1}\left\{\frac{F x^{2}}{2 m_{0}}+\frac{m_{0} c^{2}}{2} \int \frac{\eta \mathrm{d} \eta}{(1-\eta)^{2}}\right\} \\
& +\frac{m_{0} c^{2}}{2} \sum_{k=3}^{\infty} \int\left(\frac{m_{1} \eta}{F(\sqrt{1-\eta})}\right)^{k-1} \frac{\mathrm{d} \eta}{(1-\eta)^{3 / 2}}+\sum_{k=3}^{\infty} \frac{(-1)^{k} F m_{0}}{m_{1} k}\left(\frac{m_{1} x}{m_{0}}\right)^{k} .
\end{aligned}
$$


or

$$
\begin{aligned}
K(x, \eta)= & \gamma m_{0} c^{2}-F x-m_{0} c^{2}+m_{1}\left\{\frac{F x^{2}}{2 m_{0}}+\frac{m_{0} c^{2}}{2}\left[\ln (1-\eta)+\frac{1}{1-\eta}\right]\right\} \\
& +\frac{m_{0} c^{2}}{2} \sum_{k=3}^{\infty} \int\left(\frac{m_{1} \eta}{F(\sqrt{1-\eta})}\right)^{k-1} \frac{\mathrm{d} \eta}{(1-\eta)^{3 / 2}}+\sum_{k=3}^{\infty} \frac{(-1)^{k} F m_{0}}{m_{1} k}\left(\frac{m_{1} x}{m_{0}}\right)^{k},
\end{aligned}
$$

where the summation represents terms of order two or higher in the parameter $m_{1}$. The Lagrangian of the system (refLa) in terms of the variable $\eta$ is

$$
L(x, \eta)=\frac{\sqrt{\eta}}{2} \int^{\eta} \frac{K(x, \rho) \mathrm{d} \rho}{\rho \sqrt{\rho}} .
$$

So, using the above constant of motion in this expression, one gets

$$
\begin{aligned}
L(x, \eta)= & -m_{0} c^{2} \sqrt{1-\eta}+F x+m_{0} c^{2}+m_{1}\left\{-\frac{F x^{2}}{2 m_{0}}+\frac{m_{0} c^{2}}{2}\left[\frac{3 \sqrt{\eta}}{2} \ln \left|\frac{-1+\sqrt{\eta}}{1+\sqrt{\eta}}\right|-\ln (1-\eta)-1\right]\right\} \\
& +\frac{m_{0} c^{2} \sqrt{\eta}}{4} \sum_{k=3}^{\infty} \int^{\eta} \frac{\mathrm{d} \eta^{\prime}}{\eta^{\prime} \sqrt{\eta^{\prime}}} \int^{\eta^{\prime}} \frac{\left(\frac{m_{1} \rho}{F \sqrt{1-\rho}}\right)^{\mathrm{d}} \rho}{(1-\rho)^{3 / 2}}-\sum_{k=3}^{\infty} \frac{(-1)^{k} F m_{0}}{m_{1} k}\left(\frac{m_{1} x}{m_{0}}\right)^{k}
\end{aligned}
$$

The generalized linear momentum in terms of the variable $\eta$,

$$
p=\frac{2 \sqrt{\eta}}{c}\left(\frac{\partial L}{\partial \eta}\right)
$$

is given by

$$
p=\gamma m_{0} c \sqrt{\eta}+\frac{m_{1} m_{0} c^{2}}{2}\left\{\frac{1}{2 c} \ln \left|\frac{-1+\sqrt{\eta}}{1+\sqrt{\eta}}\right|+\frac{\eta}{c(1-\eta)}\right\}+\frac{m_{0} c \sqrt{\eta}}{2} \sum_{k=3}^{\infty} \frac{\partial}{\partial \eta}\left(\sqrt{\eta} \int^{\eta} \frac{\mathrm{d} \eta^{\prime}}{\eta^{\prime} \sqrt{\eta^{\prime}}} \int^{\eta^{\prime}} \frac{\left(\frac{m_{1} \rho}{F \sqrt{1-\rho}}\right)^{k-1} \mathrm{~d} \rho}{(1-\rho)^{3 / 2}}\right)
$$

Now, as one can see from this expression, even a first order in $m_{1}$ it is not possible to obtain $\eta=\eta(x, p)$ in order to get the Hamiltonian of the system. Therefore, one can not have explicitly the Hamiltonian of the system but it remains implicit through the constant of motion (31).

\subsection{Total Force Equal to Zero}

This is another important case related with neutrino mass oscillations since during the traveling of a relativistic neutrino specie, this one change to another specie,

$$
m(x)= \begin{cases}m_{1} & \text { if } 0 \leq x \leq L \\ m_{2} & \text { if } x>L\end{cases}
$$

where $m_{1}>m_{2}$. Starting from (18) with $F=0$, one gets immediately the following constant of motion

$$
\gamma m(x) v=c t e
$$

For the mass given by (36), we have

$$
\gamma_{1} m_{1} v_{1}=\gamma_{2} m_{2} v_{2}
$$

where $\gamma_{i}=\left(1-\beta_{i}^{2}\right)^{-1 / 2}$. Assuming $v_{1}$ is known (measured), the normalized velocity of the particle with mass 


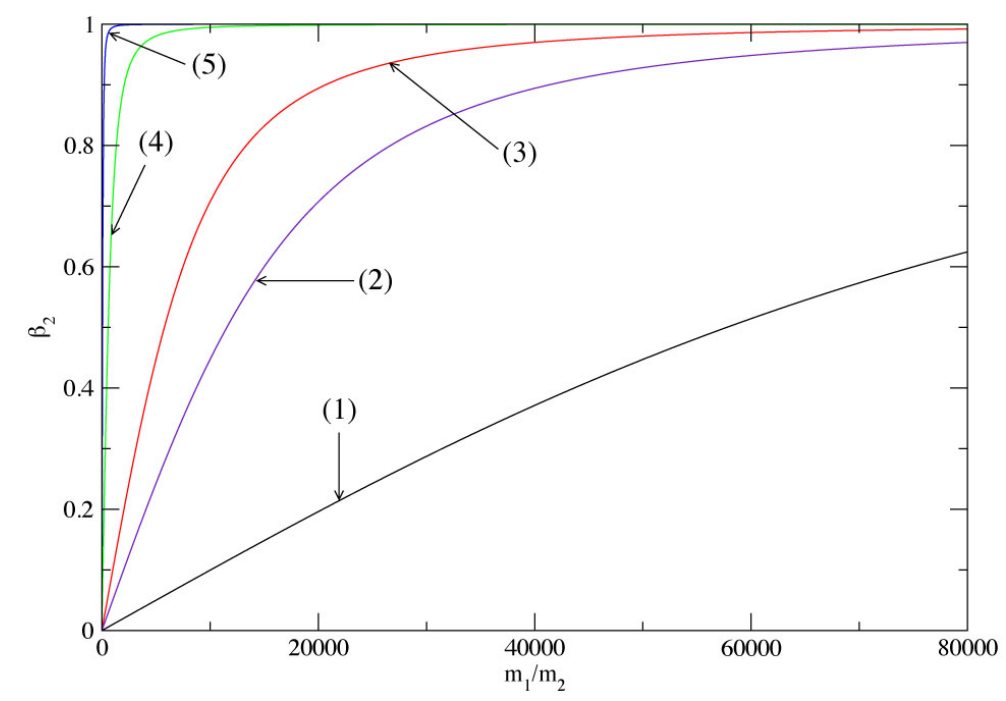

Figure 3. $\beta_{2}$ as a function of $m_{1} / m_{2}$.

$m_{2}$ is

$$
\beta_{2}=\frac{\frac{m_{1}}{m_{2}} \gamma_{1} \beta_{1}}{\sqrt{1+\frac{m_{1}^{2}}{m_{2}^{2}} \gamma_{1}^{2} \beta_{1}^{2}}} .
$$

Clearly, if $m_{1}=m_{2}$ one has that $\beta_{2}=\beta_{1}$. Figure 3 shows the behavior of $\beta_{2}$ with respect $m_{1} / m_{2}$ for $\beta_{1}=10^{-5}$ (1); $\beta_{1}=5 \times 10^{-5}$ (2); $\beta=10^{-4}$ (3); $\beta_{1}=10^{-3}$ (4); and $\beta=10^{-2}$ (5). Note from the above expression that the distance where this change of mass $(L)$ happens can not be obtained from here (one expects this point of mass change to have statistical character). In addition, for non relativistic initial velocities $\left(\beta_{1}<10^{-2}\right)$, knowing the velocity $\beta_{1}$ and measuring the normalized velocity $\beta_{2}$, the ratio $m_{1} / m_{2}$ can be determined from this plot, but for relativistic bodies $\left(\beta_{1} \geq 10^{-2}\right)$ it looks a hard matter since small variation on the measured $\beta_{2}$ implies a big variation on the ratio $m_{1} / m_{2}$. We need to point out that one can used the constant of motion (37) in the expression (11) to obtain the Lagrangian for this system, and then, the linear momentum can be calculated. However, as the above case, it is no possible to obtain explicitly $v=v(x, p)$. Therefore, it is not possible to obtain explicitly the Hamiltonian of the system.

\section{Conclusion}

We have shown that mass position depending problems for non relativistic conservative systems bring about a modification to the potential and kinetic energy terms. The constant of motion and Hamiltonian of these systems differs greatly since the generalized linear momentum depends on the position and the velocity of the body. This differences are shown with the trajectories on the spaces $(x, v)$ and $(x, p)$. For the relativistic conservative systems with mass position depending, the full integrability is not so simple in general, but we analyzed the particular case of constant force with mass linear dependence on position system. As we showed, though we can get the Lagrangian for the system, it is not possible to obtain the inverse relation $v=v(x, p)$, and therefore, the Hamiltonian of this system.

\section{References}

[1] Goldstein, H. (1950) Classical Mechanics. Addison-Wesley, Cambridge, MA.

[2] Sommerfeld, A. (1964) Lectures on Theoretical Physics. Vo. I Academic Press, Inc., New York. 
[3] Gylden, H. (1984) Astronomishe Nachrichten, 109, 11984.

[4] Meshcherskii, I.V. (1983) Astronomishe Nachrichten, 132, 93.

[5] Prieto, C. and Docobo, J.A. (1997) Astronomy and Astrophysics, 318, 657.

[6] Tuiega, C., Jasiski, J., Iwamoto, T. and Chikan, V. (2008) ACS Nano, 2, 1411-1421. http://dx.doi.org/10.1021/nn700377q

[7] Selm, W., Higazy, A. and Algradee, M. (2011) World Journal of Condensed Matter Physics, 1, 24-32. http://dx.doi.org/10.4236/wjcmp.2011.12005

[8] Takamobu, O., Kentaro, D., Koichi, N. and Akitomo, T. (2004) Physica Status Solidi (b), 241, 2744-2748. http://dx.doi.org/10.1002/pssb.200405087

[9] Sierra, L. and Lipparini, E. (1997) Europhysics Letters, 40, 667. http://dx.doi.org/10.1209/epl/i1997-00520-y

[10] Cavalcate, F.S.A., Costa Filho, R.N., Ribeiro Filho, J., De Almeida, C.A.S. and Freire, P.N. (1997) Physical Review B, 55, 1526. http://dx.doi.org/10.1103/PhysRevA.55.1526

[11] Bethe, H.A. (1986) Physical Review Letters, 56, 1305. http://dx.doi.org/10.1103/PhysRevLett.56.1305

[12] Commins, E.D. and Bucksbaum, P.A. (1983) Weak Interactions of Leptons and Quarks. Cambridge University Press, Cambridge.

[13] López, G.V. and Juárez, E.M. (2013) Journal of Modern Physics, 4, 1638-1646. http://dx.doi.org/10.4236/jmp.2013.412204

[14] Spivak, M. (2010) Physics for Mathematicians, Mechanics I. Publish or Perish Inc., USA.

[15] Von Roos, O. (1983) Physical Review B, 27, 7547. http://dx.doi.org/10.1103/PhysRevB.27.7547

[16] Li, T.L. and Kuhn, K.J. (1993) Physical Review B, 47, 12760. http://dx.doi.org/10.1103/PhysRevB.47.12760

[17] López, G.V. (2012) Partial Differential Equations of First Order and Their Application to Physics. World Scientific, Singapore.

[18] John, F. (1974) Partial Differential Equations. Springer-Verlag, New York.

[19] Kobussen, J.A. (1979) ACTA Physica Austriaca, 51, 193.

[20] Leubner, C. (1981) Physics Letters A, 86, 68-70. http://dx.doi.org/10.1016/0375-9601(81)90166-3

[21] López, G. (1996) Annals of Physics, 251, 372-383. http://dx.doi.org/10.1006/aphy.1996.0118

[22] Møller, C. (1952) Theory of Relativity. Chapter III, Oxford University Press, Oxford.

[23] Gradsteyn, I.S. and Ryzhik, I.M. (1994) Table of Integrals, Series, and Products. Academic Press, Inc., Boston. 
Scientific Research Publishing (SCIRP) is one of the largest Open Access journal publishers. It is currently publishing more than 200 open access, online, peer-reviewed journals covering a wide range of academic disciplines. SCIRP serves the worldwide academic communities and contributes to the progress and application of science with its publication.

Other selected journals from SCIRP are listed as below. Submit your manuscript to us via either submit@scirp.org or Online Submission Portal.
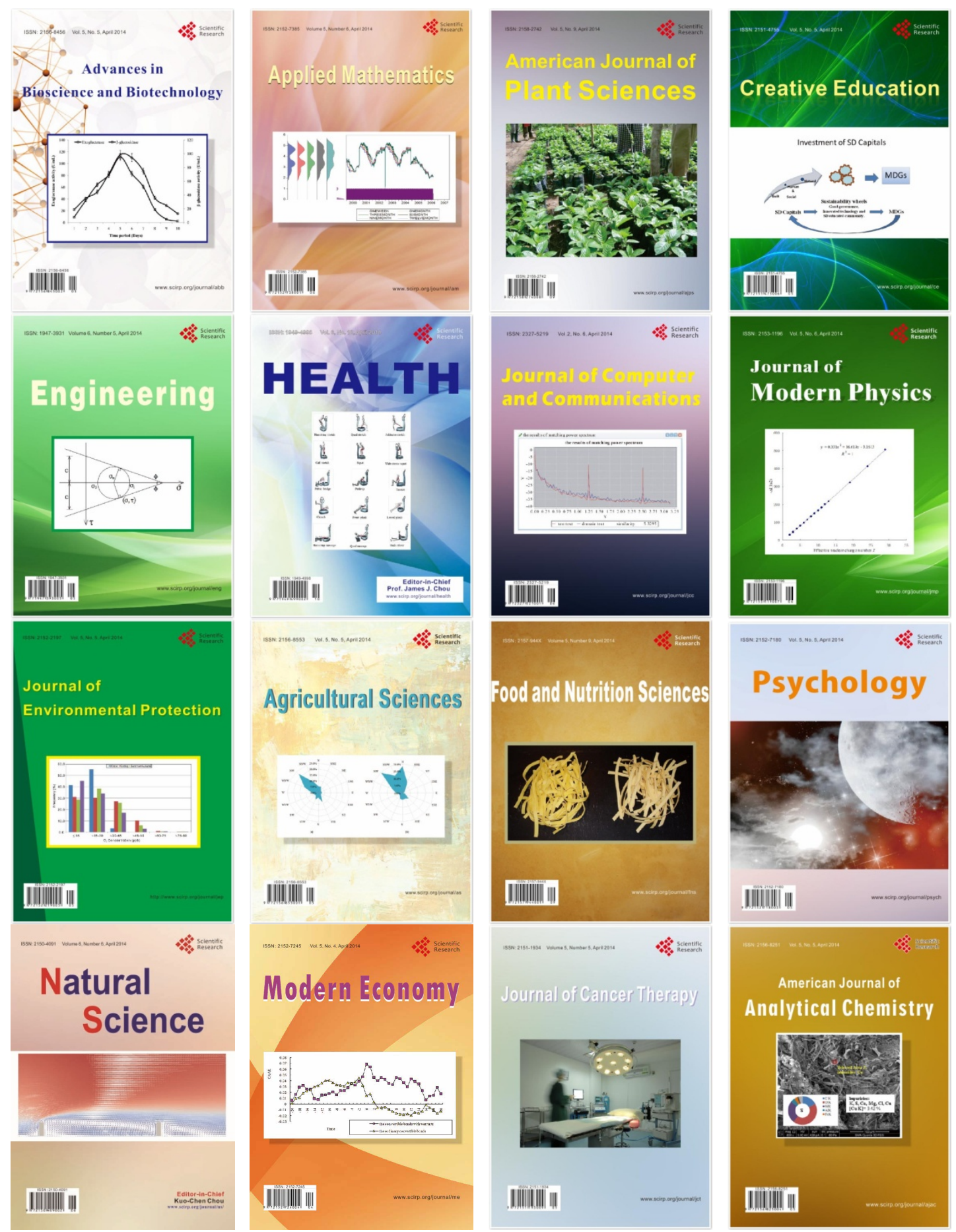